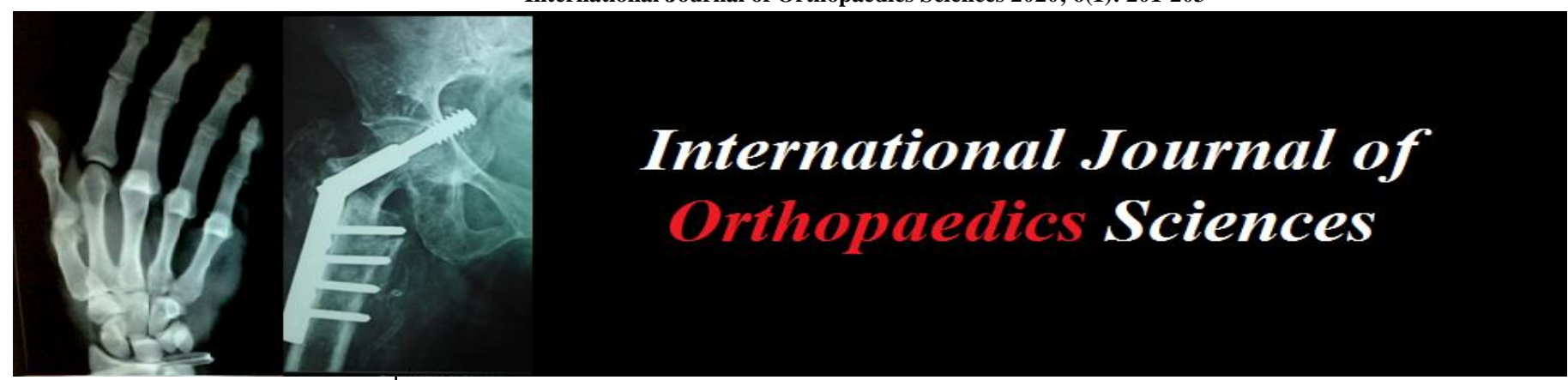

E-ISSN: 2395-1958

P-ISSN: 2706-6630

IJOS 2020; 6(1): 201-203

(C) 2020 IJOS

www.orthopaper.com

Received: 16-11-2019

Accepted: 20-12-2019

Dr. Hemeshwar Harshwardhan Professor \& Unit Head,

Department of Orthopaedics.

JLN Medical College \& Hospital,

Ajmer, Rajasthan, India

Dr. Hirdesh Kumar Saini

Post Graduate Trainee,

Department of Orthopaedics,

JLN Medical College\& Hospital,

Ajmer, Rajasthan, India

Dr. Prashant Gupta

Post Graduate Trainee,

Department of Orthopaedics,

JLN Medical College\& Hospital,

Ajmer, Rajasthan, India
Corresponding Author:

Dr. Hirdesh Kumar Saini

Post Graduate Trainee,

Department of Orthopaedics,

JLN Medical College \& Hospital

Ajmer, Rajasthan, India

\section{Assessment of clinical outcomes of PRP therapy in OA knee}

\author{
Dr. Hemeshwar Harshwardhan, Dr. Hirdesh Kumar Saini and Dr. \\ Prashant Gupta
}

DOI: https://doi.org/10.22271/ortho.2020.v6.i1d.1862

\section{Abstract}

PRP is an autologous mixture of highly concentrated platelets and associated growth factors and other bioactive components produced by centrifugal separation of whole blood used in OA knee. The purpose of the study was to evaluate the role of PRP in management of OA knee and its clinical outcome in term of decrement in WOMAC and VAS scores up to 12 months follow up. 100 patients with 150 knees (65 female, 35 male) with Kellgren-Lawrence grade 1, 2, 3 and 4 aged between 50 to 72 years evaluated between November 2017 to October 2019. All patients were treated with $4 \mathrm{ml}$ of intra-articular PRP injection at day 1 and at the end of 1st, 3rd, 6th and 12 months and evaluated on each visit with WOMAC and VAS scores. Decrement in WOMAC score for KL grade $1(44.26 \%)$, grade $2(31.89 \%)$, grade 3 (29.7\%), KL grade $4(6.57 \%)$ at the end of 12 months follow up. Decrement in VAS score for KL grade1 $(57.63 \%)$, KL grade $2(42.27 \%)$, KL grade $3(28.47 \%)$, KL grade $4(3.94 \%)$ at the end of 12 months follow up. Decrement in WOMAC and VAS score were statically significant with p value $(<0.05)$ at 12 months follow up for K.L. grade 1, 2 and 3. Decrement was not statically significant for K.L. grade 4. Our study results shows that PRP therapy is minimal invasive and effective in reduction of knee pain and stiffness and improvement in quality of life only in patients with early stages of osteoarthritis knee.

Keywords: Platelet rich plasma, WOMAC, VAS

\section{Introduction}

Knee osteoarthritis (OA) is the most common articular disease. Different methods are used to alleviate the symptoms of patients with knee OA, including analgesics, physical therapy, exercise prescription, and intra-articular injections (glucocorticoids, hyaluronic acid [HA], etc). Including the use of platelet-rich plasma (PRP), an autologous growth factor treatment.

\section{Aims and Objectives}

To evaluate clinical and functional outcome of PRP therapy in knee osteoarthritis.

\section{Material and Methods}

\section{Source of data}

We prospectively analysed 100 patients (150knees) of osteoarthritis knee coming to the orthopaedics department at Jawahar Lal Nehru Medical College, Ajmer, Rajasthan between November 2017 to october2019 fulfilling exclusion and inclusion criteria.

\section{Inclusion criteria}

Patients (50-72 years) with clinical symptoms of osteoarthrosis knee and the radiologic signs of knee with OA Grade 1, 2, and 3 and 4 according to Kallgren and Lawrence classification for more than 1 year with failure to reduce symptoms after analgesics.

\section{Exclusion criteria}

Infection in and around knee, history of severe trauma to the knee, Immune compromise state, collagen vascular diseases, any malignant disorders, clotting disorders, taking anticoagulant and antiplatelet medications, history of intraarticular knee injections of corticosteroids, abnormal blood cellular counts. 


\section{Statistical analysis}

Data were analysed using GraphPad Software (Inc.7825 Fay Avenue, Suite 230 La Jolla, CA 92037 USA). Data from Kallgren-Lawrence grade and WOMAC score/VAS score, MRI cartilage thickness were evaluated by the paired $t$ test. $P$ value $<0.05$ was accepted as statistically significant for all tests.

\section{Outcome Analysis}

Patients were evaluated using Western Ontario and McMaster Universities Arthritis Index (WOMAC) and Visual Analogue Score (VAS) at day 1 , at the end of $1 \mathrm{st}$, 3rd, 6th and 12th month on follow up.

\section{Results and Observations}

- Mean age of patients under study was 63.21 years. 100 patients (65 femlaes, 35 males) with 150 knees were evaluated.

Table 1: Mean age of patients under study was 63.21 years

\begin{tabular}{|c|c|c|}
\hline Sex & No. of Patients & No of knees \\
\hline Male & 35 & 52 \\
\hline Female & 65 & 98 \\
\hline
\end{tabular}

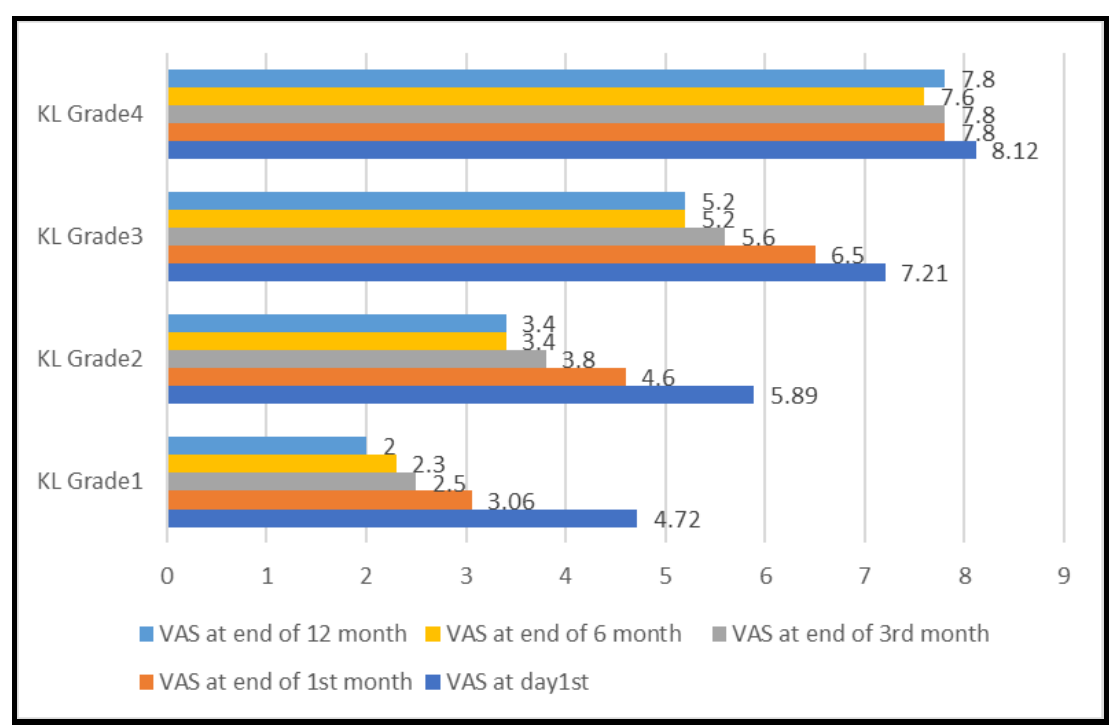

Chart 1: Chart showing VAS scoring as per KL grade of OA knee

Table 2: Mean of total WOMAC score as per K.L. grades of OA knee

\begin{tabular}{|c|c|c|c|c|c|}
\hline K.L. Grade & $\begin{array}{c}\text { Mean of Total } \\
\text { WOMAC score } \\
\text { at day 1 st }\end{array}$ & $\begin{array}{c}\text { Mean of Total } \\
\text { WOMAC score at } \\
\text { end of 1st month }\end{array}$ & $\begin{array}{c}\text { Mean of Total } \\
\text { WOMAC score at } \\
\text { end of 3rd month }\end{array}$ & $\begin{array}{c}\text { Mean of Total } \\
\text { WOMAC score at } \\
\text { end of 6th month }\end{array}$ & $\begin{array}{c}\text { Mean of Total } \\
\text { WOMAC score at } \\
\text { end of 12th month }\end{array}$ \\
\hline KL Grade 1 & 30.86 & 25.06 & 20.7 & 18.4 & 17.2 \\
\hline KL Grade 2 & 41.71 & 36.8 & 30.4 & 28.4 & 28.4 \\
\hline KL Grade 3 & 52.35 & 45.8 & 40.6 & 36.3 & 36.8 \\
\hline KL Grade 4 & 62.40 & 59.1 & 57.4 & 58.1 & 58.3 \\
\hline
\end{tabular}

- In our study only KL grade 1, 2 and 3 showed statically significant improvement ( $\mathrm{p}$ value $<0.05$ ) in WOMAC and VAS score at 12 month follow up.

- For KL grade 4 OA there is no statically significant improvement in WOMAC and VAS score.

- No major complications were noted in our study which is comparable with other study reports and signifies the safety profile of autologous PRP intra-articular injections. No infection, fever, or other major adverse events occurred among study subjects. 7 patients had mild effusion and pain after the injections, which persisted for not more than 4 days.

- Mean platelet concentration was about 5-fold increase in mean platelet concentration.

\section{Discussion}

Platelet-rich plasma (PRP) is an autologous biologic treatment including patients own plasma, containing growth factors released from platelets and endogenous fibrin scaffold. The rationale for the use of PRP is to stimulate the natural healing cascade and tissue regeneration by a "supra-physiologic" release of platelet-derived factors directly at the site of treatment ${ }^{[1]}$.
The purpose of this study was to investigate the effectiveness of intra-articular PRP injections in active patients with symptomatic knee OA in terms of diminishing pain, improving quality of life, and returning to previous activities. All patients showed significant improvement in WOMAC score at 1, 3, 6 and 12 months ( $P$ value $<0.05$ ) except grade 4 $\mathrm{OA}$ at 12 month follow up. All patients showed significant improvement in VAS score at 1, 3, 6 and 12 months ( $P$ value $<0.05$ ) except grade $4 \mathrm{OA}$ at 12 month follow up. It concluded that PRP injections can represent a valuable treatment in patients with early OA knee (K.L. grade 1, 2, 3) in long term follow up.

Our study results are comparable with other conducted studies by Sampson S at al. ${ }^{[5]}$ (2010), Filardo G et al. ${ }^{[6]}$ (2011), Dhillon M et al. ${ }^{[8]}$ (2011) and other studies.

The main limitation of our study was that we did not include a control group and did not included radiological investigation like MRI to evaluate effect on cartilage.

\section{Conclusion}

The findings of the current study suggest that PRP is minimally invasive, safe and cost-effective treatment for early grades of osteoarthritis in terms of diminishing pain, 
improving quality of life, and returning to previous activities. However, more large studies needed to evaluate long term outcome of PRP therapy.

\section{References}

1. Seyed Ahmad Raeissadat, Seyed MR, Hossein Hassanabadi, Mohammad F. Knee Osteoarthritis Injection Choices: Platelet- Rich Plasma (PRP) Versus Hyaluronic Acid (A one-year randomized clinical trial) Published online 2015, 7. doi: 10.4137/CMAMD.S17894

2. Meheux CJ, McCulloch PC, Lintner DM, Varner KE, Harris JD. Efficacy of Intra-articular Platelet-Rich Plasma Injections in Knee Osteoarthritis: A Systematic Review. Arthroscopy. 2016; 32(3):495-505. doi: 10.1016/j.arthro.2015.08.005. Epub 2015 Oct 1.

3. Prashant Maheshwari, Hemeshwar Harshwardhan. Platelet rich plasma for the treatment of osteoarthrosis knee. http://dx.doi.org/10.18203/issn.2455-4510. Int. J Res Orthop. 20170781

4. Spaková T, Rosocha J, Lacko M, Harvanová D, Gharaibeh A. Treatment of knee joint osteoarthritis with autologous platelet-rich plasma in comparison with hyaluronic acid. Am J Phys Med Rehabil. 2012; 91(5):411-7. doi: 10.1097/PHM.0b013e3182aab72.

5. Sampson S, Reed M, Silvers H, Meng M, Mandelbaum B. Injection of platelet-rich plasma in patients with primary and secondary knee osteoarthritis: a pilot study. American Journal of Physical Medicine \& Rehabilitation. 2010; 89(12):961-9.

6. Filardo G, Kon E, Ruiz MT, Vaccaro F, Guitaldi R, Di Martino A et al. Platelet-rich plasma intra-articular injections for cartilage degeneration and osteoarthritis: single- versus double-spinning approach. Knee Surg Sports Traumatol Arthrosc. 2011, 2012; 20(10):2082-91. 